\title{
INTELIGENCIA TÁCTICA
}

\author{
José Antonio Sainz de la Peña ${ }^{1}$ \\ UNISCI
}

\begin{abstract}
Resumen:
Existe una notable diferencia entre la batalla propiamente dicha y las acciones militares que la preceden y la siguen. El nivel táctico se organiza para ganar los combates y batallas y tiene unas características propias que le diferencian claramente, al menos desde el punto de vista teórico, de los niveles estratégico y operativo. A nivel táctico, la producción de inteligencia sigue el mismo patrón que en los niveles estratégico y operativo, pero adaptándose el ciclo a la medida del escalón. Sus principales características son la urgencia y la limitación de medios que hacen que, muchas veces, se trabaje con la información bruta o semielaborada. Su ventaja es el contacto directo con la situación. En los conflictos asimétricos, la Inteligencia Táctica seguirá contando con su gran ventaja, el "contacto" con la realidad, y también con su gran inconveniente, la falta de tiempo. A lo que hay que añadir el impacto de las nuevas tecnologías en la Inteligencia Táctica y sus posibles consecuencias negativas: el coste de los sistemas, la fragilidad de los mismos, la sobreinformación resultante y los daños a la cadena de mando. La sobreinformación puede producir indecisión, errores de interpretación e incluso abrir el camino a la indisciplina en la cadena de mando en sistemas NEC.
\end{abstract}

Palabras clave: Inteligencia Táctica, estrategia, batallas, nivel operativo, conflictos asimétricos, NEC.

Title in English: "Tactical Intelligence”.

\begin{abstract}
:
The article explains that a notable difference exists between the battle and the military actions that precede and follow it. The tactical level is organised to win the fights and battles, has its own characteristics that distinguish it clearly, at least from a theoretical standpoint, from the strategic and operational levels. At the tactical level, the production of intelligence follows the same pattern as in strategic and operational levels. But its main futures that differentiate it from other levels of intelligence are urgency and limited means. All this frequently implies working with raw or semiprocessed information. The impact of new technologies has also to be mentioned. Information overload can cause indecision, misunderstanding and even would pave the way for indiscipline in the chain of command.
\end{abstract}

Keywords: Tactical Intelligence, Strategy, Battle, Operational level, Asymmetric conflicts, NCW.

Copyright (C) UNISCI, 2012.

Las opiniones expresadas en estos artículos son propias de sus autores, y no reflejan necesariamente la opinión de UNISCI. The views expressed in these articles are those of the authors, and do not necessarily reflect the views of UNISCI.

\footnotetext{
${ }^{1}$ José Antonio Sainz de la Peña es investigador senior de UNISCI e-mail: esedelape@hotmail.com. http://dx.doi.org/10.5209/rev_UNIS.2012.n28.3840u
} 


\section{Introducción}

$\mathrm{Si}$ alguien entra en Internet buscando INTELIGENCIA TÁCTICA encontrará, sorprendentemente, que la mayoría de las páginas halladas se referirán a entrenadores o jugadores deportivos, fundamentalmente de fútbol o rugby, de los que se alaba su "inteligencia táctica".

Este documento tiene un objetivo muy diferente, menos divertido y limitado al ámbito militar. Por ello, es preciso, primero, definir o delimitar lo que entendemos por "inteligencia táctica", expresión que une dos palabras que, también ambas, necesitan aclaración.

Los países anglosajones han utilizado tradicionalmente la palabra "inteligencia" para cierto tipo de conocimiento utilitario para el que los latinos han empleado "información"; el concepto subyacente es idéntico. Para los últimos, la "información" es el resultado de analizar y procesar "noticias" mientras que para los primeros la "inteligencia" es la consecuencia de analizar y procesar "informaciones". En ambos casos, lo que se pretende es ayudar a tomar decisiones correctas.

Según el Diccionario de la Real Academia Española (DRAE) inteligencia es "facultad intelectiva", "capacidad de entender o comprender" o "conocimiento o comprensión". Es decir, que en el idioma español, la inteligencia poco o nada tiene que ver con lo que en el lenguaje militar de hoy recibe el nombre de "inteligencia"

Por el contrario, el DRAE define la "información" como "comunicación o adquisición de conocimientos que permiten ampliar o precisar los que se poseen sobre una materia determinada" (acepción $5^{\mathrm{a}}$ ). Esta definición se corresponde, casi perfectamente, con el concepto anglosajón de "inteligencia". Este concepto fue adoptado por la OTAN y, en consecuencia, por todos los países miembros de la Alianza Atlántica. "La inteligencia es el producto resultante de procesar la información sobre naciones extranjeras, fuerzas hostiles o potencialmente hostiles o elementos o áreas de operaciones posibles o actuales" (Glosario de Términos de la OTAN- AAP-6; 2009).

En España, la "inteligencia” entró en el lenguaje militar desde los Estados Unidos a través, primero, de la Marina y, después, del Ejército del Aire. Posteriormente, el Ejército de Tierra aceptó e impuso el término a finales de los años 80 que, por último, fue consagrado oficialmente, por completo, cuando el antiguo CESID, Centro de Información, fue rebautizado como CNI, Centro de Inteligencia.

Para la Doctrina militar del Ejército de Tierra "La inteligencia es, en un sentido amplio, el resultado de la integración e interpretación de nuestros conocimientos sobre el terreno, meteorología, población, actividades, capacidades e intenciones de un enemigo presente o potencial" (Doctrina C-2-001-julio 1998)

Por su parte, el Manual utilizado por el ejército estadounidense en sus cursos para oficiales de otros ejércitos americanos, en la "Escuela de las Américas", describe la inteligencia militar como "los conocimientos adquiridos a través de los esfuerzos de búsqueda, evaluación e interpretación de toda la información disponible que tiene que ver con un enemigo real o hipotético o con ciertas áreas de operaciones, incluyendo las condiciones meteorológicas y el terreno". La similitud de las definiciones es extraordinaria y supone un origen común. 
Sin embargo, conviene recordar que, si bien el uso de la palabra "inteligencia", en el sentido indicado, es casi universal, el Ejército francés sigue empleando el término tradicional de "renseignement" (información), incluso en la versión en lengua francesa del Glosario OTAN, que es oficial junto con la versión inglesa, que los británicos utilizan ambas palabras y que las Naciones Unidas, en sus intervenciones tipo "cascos azules", no usan "inteligencia" sino"información militar".

En España la guerra está perdida; la "inteligencia" la ha ganado, la "información" ha perdido. Seguiremos, por lo tanto, hablando de "inteligencia", aunque resulte más normal pensar que un jefe militar diga a su Jefe de Estado Mayor:

- Fulano ¡Infórmeme de la situación!

y no que le diga: ¡Inteligéncieme!

\section{Los niveles de decisión}

\subsection{Los niveles políticos}

En todo Estado, el poder político, para conseguir los objetivos nacionales, se apoya, básicamente, en un trípode de Instrumentos: la diplomacia, la economía y la fuerza militar. De esta realidad nace la "Pirámide de Estrategias" o modo de actuar en los diversos niveles de decisión para alcanzar los objetivos fijados por el poder político.

El vértice de la "Pirámide" es la "Estrategia Nacional" o "Gran Estrategia", que es el nivel donde se determinan los objetivos, se asignan recursos para lograrlos y se coordinan los instrumentos del poder nacional.

A continuación, vienen las "Estrategias Departamentales", por las que cada departamento del Gobierno, en especial los de Asuntos Exteriores, de Economía y de Defensa, desarrolla y planifica sus modalidades de acción para obtener los resultados marcados en la "Estrategia Nacional"; entre aquellas, está la "Estrategia Militar General".

En esta "Estrategia Militar" se enlazan las decisiones políticas con las posibilidades militares, se establecen prioridades, se reparten medios y se determina cómo deber organizarse y emplearse la fuerza militar. Es interesante hacer notar que en la "Estrategia Militar General", se pueden lograr los objetivos tanto por el uso de la fuerza como, únicamente, por la amenaza de su empleo.

Descendiendo por la "Pirámide", después se encuentran los niveles de decisión propios de las Fuerzas Armadas.

\subsection{Los niveles militares}

Los tratadistas militares clásicos siempre distinguieron dos niveles dentro de lo que se conoce como "Arte de la Guerra": el "Estratégico" y el "Táctico". Al primero, le correspondía la dirección general de la guerra; al segundo, la ejecución. Sin embargo, tanto para Clausewitz como para Jomini, los dos grandes teóricos de la guerra en el siglo XIX, existía una clara diferencia entre la batalla propiamente dicha y las acciones militares que la preceden y la 
siguen. Antes de la batalla, estaba su concepción y su preparación; después, la explotación o la retirada. Todas esas acciones necesitaban un tratamiento teórico distinto.

De esta distinción nació una rama o nivel del Arte de la Guerra situada entre la Estrategia y la Táctica, a caballo entre ellas y distinta de ambas, a la que se denominó "Gran Táctica", "Estrategia Militar", o "Estrategia Operacional", la estrategia propia del "Nivel Operacional".

Las naciones occidentales y, más tarde, la OTAN, no reconocieron en sus Doctrinas Militares el Nivel Operacional hasta después de la Segunda Guerra Mundial. En cambio, las Fuerzas Armadas soviéticas desarrollaron, desde los años 20 del siglo pasado, el concepto de "Arte Operacional", distinguiendo así tres niveles: el Estratégico, el Operacional y el Táctico. Frunze decía que la Estrategia sólo indica la dirección general, la Operativa concibe las operaciones y la Táctica, las materializa con acciones. Los ejércitos occidentales sólo vieron en el "Arte Operacional" soviético un escalón de mando intermedio, necesario para poder manejar las grandes masas humanas del Ejército Rojo, maniobrando en la gigantesca extensión del imperio soviético. Sin embargo, el concepto es más profundo y se basa en el emparejamiento Objetivo Estratégico - Arte Operacional.

El nivel operacional está hoy reconocido por todos los ejércitos como el nivel en el cual se conciben y ejecutan las operaciones y campañas, en el que se planifican, conducen y sostienen las operaciones para alcanzar los objetivos estratégicos, fijados por el nivel estratégico, en un determinado Teatro o Zona de Operaciones,. Es un nivel militar teóricamente distinto del Estratégico y del Táctico. El Nivel Operacional es el de la dirección de las operaciones militares y del despliegue y empleo de las fuerzas para alcanzar los objetivos asignados a las unidades militares por el nivel estratégico.

En resumen, el nivel táctico se organiza para ganar los combates y batallas que constituyen las operaciones. Las operaciones se organizan para ganar las campañas y éstas para ganar la guerra.

No obstante, aunque la distinción teórica entre los tres niveles es clara, en la práctica puede haber interferencias mutuas. Además, si bien los niveles se corresponden, en principio, con el tamaño o entidad de la unidad militar ejecutante (en el estratégico, las Grandes Unidades Superiores; en el operacional, las Grandes Unidades Inferiores; en el táctico, las Pequeñas Unidades), lo verdaderamente importante no es el tamaño de la unidad sino el objetivo buscado. Una Pequeña Unidad puede actuar en el nivel operativo si busca lograr un objetivo estratégico ${ }^{2}$.

\subsection{Los niveles de inteligencia}

Todos los niveles de decisión y ejecución antes citados necesitan conocimientos que les permitan tomar decisiones acertadas, es decir, un sistema o método de ayuda a la decisión. En otras palabras, cada nivel estratégico o táctico necesita una "inteligencia" adecuada a su nivel.

Por ello, existe en cada nación un servicio o sistema de inteligencia nacional al más alto nivel e inteligencias departamentales en cada respectivo campo de competencias. La inteligencia que aquí nos interesa es la Militar y, dentro de ésta, las inteligencias estratégica,

\footnotetext{
${ }^{2}$ Militarmente se suelen llamar Grandes Unidades Inferiores a las Divisiones y Brigadas, Grandes Unidades Superiores a las de mayor entidad a la División y Pequeñas Unidades a las menores a la Brigada.
} 
operacional y táctica, todas ellas organizadas y adaptadas para satisfacer las necesidades de conocimiento de cada nivel. Así como existe una "Pirámide de Estrategias", existe también una "Pirámide de Inteligencias".

Entre las dos "Pirámides", hay una diferencia característica. En la de Estrategia, cada nivel es el "sirviente" del superior y el "amo" del inferior; el flujo entre niveles es unidireccional y siempre de arriba abajo. En la segunda "Pirámide", cada nivel es sirviente del anterior pero también le demanda conocimientos; el flujo es bidireccional. (Figura 1)

La "Pirámide de Inteligencias" es en realidad un "sistema", en el sentido matemático de la palabra, cuyos elementos o componentes son los diferentes niveles de inteligencia (que a su vez son subsistemas) y las relaciones que los unen son los lazos de petición de información y de difusión de la misma. Por otra parte, y por las características propias de la inteligencia, las áreas de trabajo de esos subsistemas no son compartimentos estancos sino que se superponen parcialmente.

NIVEL

POLITICO

DEPARTAMENTAL

ESTRATÉGICO

OPERACIONAL
ESTRATEGIAS

INTELIGENCIA
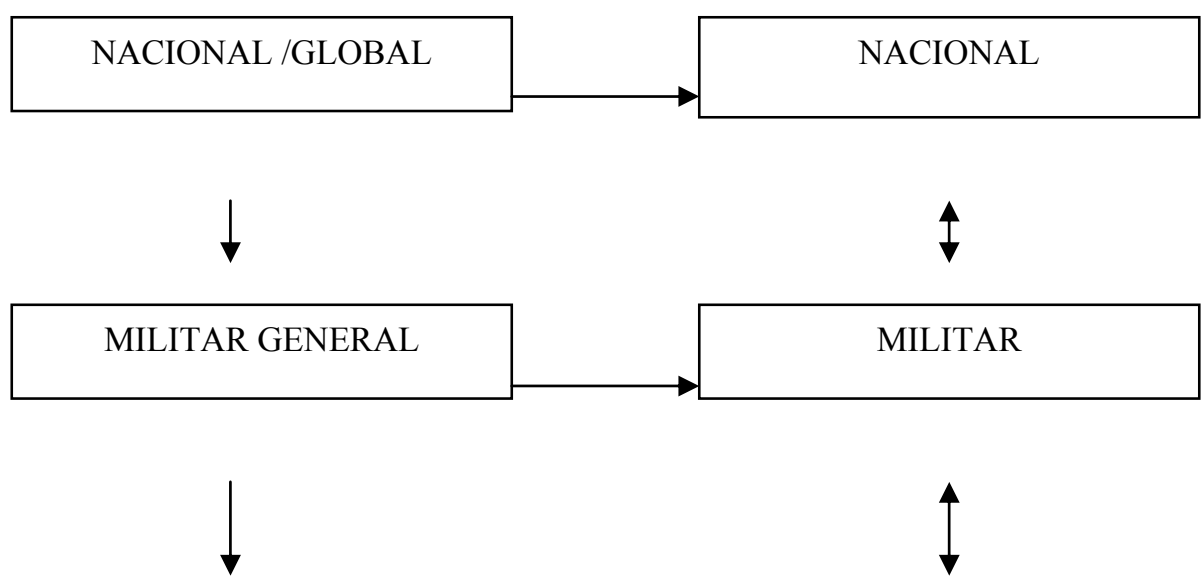

ESTRATEGIA MILITAR
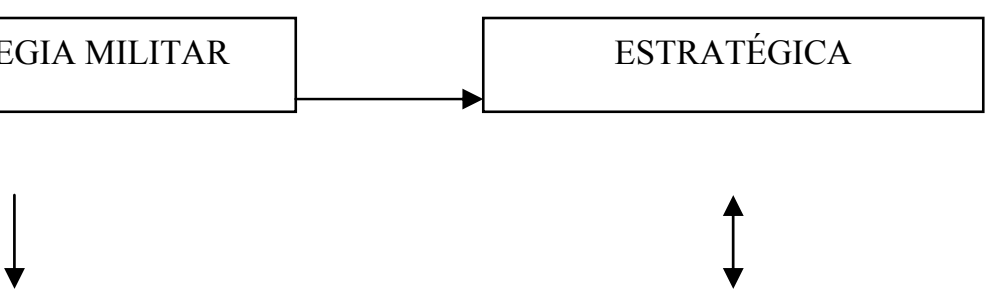

ARTE OPERATIVO

OPERACIONAL 
TÁCTICO

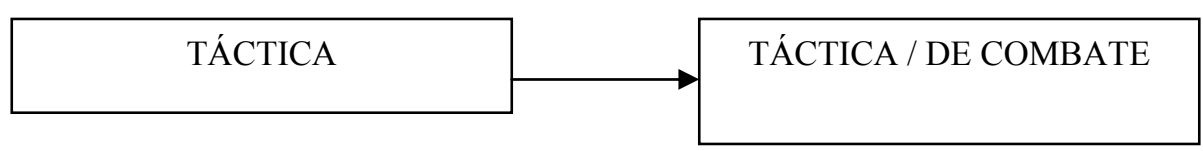

\subsection{Tipos de Inteligencia}

En todos los niveles de la Pirámide hay tres tipos de inteligencia: Básica, Actual y Predictiva.

La Inteligencia Básica es el conjunto de documentos sobre asuntos que interesan, que tienen validez durante un tiempo, más o menos prolongado, que se conservan mientras permanece esa validez, que se actualizan constantemente y que se utilizan como fuente para el planeamiento de acciones y como comparación con nueva inteligencia o información. Es una "Base de Datos". Ejemplo de inteligencia básica que están en poder de los Estados Mayores son la colección de planos actualizados sobre áreas geográficas o el documento llamado ORBAT (Orden de Batalla) sobre un adversario real o potencial, en el que figuran su historia, su doctrina, su organización, su material, sus mandos, su despliegue y hasta su psicología y uniformidad.

La Inteligencia Actual es la que se refiere a la situación en el momento actual y a los sucesos que se está desarrollando. Esta Inteligencia sirve, además, para actualizar la Básica.

La Inteligencia Predictiva, llamada también Estimativa o Especulativa, es aquella que, partiendo de la Básica y de la Actual, intenta predecir la posible actuación del adversario.

\subsection{La decisión}

En cada escalón de mando de la organización militar, el Jefe toma sus decisiones en función de dos factores principales: la Misión y la Situación.

La Misión es lo que se ha ordenado hacer; es imperativa y hay que cumplirla. Es el factor principal.

La Situación está compuesta por un conjunto de factores que analizados e integrados, siempre bajo el prisma del cumplimiento de la Misión, dirá cómo ésta se puede cumplir. El primer factor de la Situación que debe analizar el Jefe son los Medios con los que cuenta. Este factor es conocido y, por ello, no necesita esfuerzo de "inteligencia". Todo lo más, la puesta al día de estadillos de personal y material y alguna revista de los equipos y su puesta a punto.

Después viene el factor Terreno, factor casi invariable en sus formas pero que, normalmente, necesita ser actualizado por la existencia de nuevas construcciones o destrucciones, de nuevas vías de comunicación, etc. El análisis del Terreno incluye la Meteorología, ya que ésta puede modificar el terreno, impidiendo, facilitando o modificando su utilización.

Pero el factor de mayor importancia de la Situación, al estudio del cual tiene que dedicar la inteligencia el mayor trabajo, es el Enemigo. Conocer qué es - su entidad, su despliegue, su fuerza y material-, qué puede hacer y qué quiere hacer es la mayor preocupación del Jefe.

A estos factores tradicionales se añade actualmente y, en distintos grados, según el escalón militar, el Ambiente en el cual se va a desarrollar la acción, cuyo subfactor más 
importante es la población civil de la zona (número, actitud, ideología, grupos políticos o paramilitares etc.).

El órgano de Inteligencia de cada escalón tendrá que suministrar al Jefe datos valorados e interpretados de todos los factores de la decisión, excepto el análisis de los medios propios. Para los demás factores se basará en la Inteligencia Básica disponible, en su propio esfuerzo de Inteligencia Actual y en la Inteligencia de otros escalones. Para conseguir esto último, hará peticiones al escalón superior y a los de su mismo nivel y dará órdenes a los inferiores.

\section{El nivel Táctico}

\subsection{Características del nivel táctico}

El DRAE define la táctica como el conjunto de reglas a las que se ajustan en su ejecución las operaciones militares.

Para los militares, la Táctica es un "Arte"; el arte de desplegar y mover las tropas en el campo de batalla, con orden y recíproca protección, para alcanzar objetivos militares.

También es : el arte de emplear unidades militares, de Tierra, Mar, Aire y Especiales para realizar actividades que contribuyen al éxito de la operación (ejército británico) ; el de combinar, en operaciones, las acciones de todos los medios militares para alcanzar los objetivos asignados por al estrategia operacional (ejército francés); o el del empleo de unidades de combate, teniendo en cuenta su disposición ordenada y la maniobra de las unidades, la relación de unas con otras, el terreno y el enemigo para transformar el potencial militar en batallas y combates victoriosos (ejército norteamericano).

El nivel táctico tiene unas características propias que le diferencian claramente, al menos desde el punto de vista teórico, de los niveles estratégico y operativo. Aún así, en la práctica puede haber solapes e interferencias.

- En primer lugar, su visión del problema militar no es global, ni siquiera amplia, sino, por el contrario, muy reducida. El Terreno lo estudia hasta el más mínimo detalle, pero ese estudio se limita a su zona de acción asignada, a su "compartimiento"; todo lo más, tiene en cuenta las zonas situadas a sus flancos.

- La situación que le interesa es la actual, no la pasada.Tampoco hace hipótesis de futuro.

- El enemigo no es el ejército adversario, sino las unidades de ese ejército que tiene enfrente.

- No se pregunta por las intenciones enemigas, sino por las capacidades de sus medios, de lo que deduce sus posibilidades reales.

- El factor preponderante es el militar y los demás factores del Ambiente son despreciables o secundarios.

- Los objetivos a alcanzar son puramente militares y los recibe del nivel operativo. 
- Su punto de vista es, fundamentalmente, terrestre, aunque hoy la batalla sea siempre, en realidad, aeroterrestre y muchas veces aeronaval.

- Las fuerzas militares que participan en el combate son o Pequeñas Unidades o Grandes Unidades menores (Brigadas, División).

- Así como el espacio en que se mueve es limitado, el tiempo de que se dispone es siempre escaso, lo que implica decisiones rápidas y, por ello, arriesgadas.

Finalmente, así como en tiempos de Napoleón, la Táctica era un "Arte sencillo, todo ejecución", hoy día, la Táctica es, cada vez, más complicada.

\subsection{Características de la Inteligencia Táctica}

En primer lugar hay que subrayar que la Inteligencia Táctica es una "Función de Combate" de carácter permanente, como lo son la maniobra o el apoyo de fuegos o la logística o la movilidad o el ejercicio del mando, que no es un fin en si misma y que no está limitada a algunos especialistas. Todo combatiente debe participar en ella.

La Inteligencia Táctica se define como la Inteligencia necesaria para el planeamiento y la ejecución de operaciones tácticas, teniendo en cuenta el terreno, la meteorología y el enemigo. A veces, se la denomina "Inteligencia de Combate".

También puede definírsela, de un modo positivo, como la inteligencia dirigida a responder las necesidades de los Mandos militares en campaña, para mantener la disponibilidad de las fuerzas para el combate y ayudar al planeamiento y ejecución de operaciones de combate. $\mathrm{O}$ de manera negativa como el arte y ciencia de determinar lo que el adversario está haciendo o puede hacer para evitar el cumplimiento de la misión.

Parafraseando la definición de Inteligencia Estratégica de S. Kent, podemos decir que la Inteligencia Táctica es "Inteligencia positiva, extranjera, a nivel táctico". Es positiva porque excluye la contrainteligencia y la seguridad. Es un concepto positivo, no negativo como la contrainteligencia, aunque, en ocasiones, comparta con ésta medios y métodos y no es seguridad aunque, indirectamente, la proporcione. Es extranjera porque sólo se ocupa del enemigo exterior. Es de nivel táctico, ya que no considera ni lo estratégico ni lo operacional. Táctico.

Sus características se deducen y se corresponden con las características del Nivel

- La más determinante de todas es la "inmediatez". La premura obliga a una elaboración y difusión rápida de inteligencia e, incluso, a veces, a difundir información bruta, sin elaborar, en tiempo real.

- La Inteligencia Básica necesaria para el cumplimiento de su misión la recibirá siempre de escalones superiores. La Predictiva no entra ni en el campo de su competencia ni en sus posibilidades. Por en contrario, la Inteligencia Actual es su dominio privilegiado. $\mathrm{Su}$ contacto con la realidad de la Situación hace que pueda recoger información múltiple y reciente, algo que no está al alcance de los niveles superiores. 
- Territorialmente, cada unidad tiene una "Zona de Responsabilidad" táctica y unas "Zonas de Interés" que son las de responsabilidad de las unidades vecinas. Este esquema se repite en el campo de la inteligencia, en el que cada unidad táctica tiene su "Zona de Responsabilidad" de inteligencia y sus "Zonas de Interés". El campo en el que trabaja la Inteligencia Táctica es limitado y se reduce, principalmente, a su "compartimiento".

- El Terreno lo estudia con minuciosidad, descendiendo al detalle y actualizando los documentos y datos, fundamentalmente mapas, recibidos del escalón superior o procedentes de la Inteligencia Básica.

- Los datos meteorológicos le llegarán de los servicios meteorológicos especializados. Sin embargo, en ocasiones, puede recibir datos parciales de elementos propios, como de unidades de helicópteros o artilleras. En principio, solamente tendrá que considerar su influencia en las operaciones, integrando esos datos en el estudio del terreno, en particular, en sus condiciones de viabilidad.

- La Inteligencia Táctica sólo se interesa en los aspectos de la situación que pueden influir directamente en el combate. Por lo tanto, prescinde, casi siempre, de los aspectos no específicamente militares.

- La Inteligencia Táctica no es un sistema cerrado e independiente. Es un subsistema del sistema general de Inteligencia Nacional y se integra en él a través del subsistema de Inteligencia Operacional.

- Por sus limitaciones de tiempo, espacio y medios, cada escalón de la Inteligencia Táctica tiene siempre muy en cuenta las valoraciones e interpretaciones del escalón superior al suyo.

- La necesidad prioritaria de la Inteligencia Táctica es el estudio del enemigo que tiene enfrente. El conocimiento de su despliegue, de sus actividades, de sus medios humanos y materiales es la parte más importante de su trabajo.

- En la Inteligencia Táctica, cobra todo su valor la consideración de la Inteligencia como conocimiento utilitario. Todo lo que no interesa para el combate, no sirve.

- En el nivel táctico, al tratarse de unidades militares de pequeña identidad, los medios orgánicos de inteligencia, tanto técnicos como humanos, siempre son escasos.

\section{Los principios de la Inteligencia}

La OTAN admite ocho principios fundamentales en los que debe basarse la inteligencia militar, en todos sus niveles y escalones. Esos principios los recogen todos los países miembros de la Alianza.

Los "Principios" son: Control Centralizado, Oportunidad, Explotación Sistemática de las Fuentes, Objetividad, Accesibilidad, Capacidad de Respuesta, Protección de las Fuentes, Revisión Continua y Comunicación. 
De los ocho Principios, algunos son tan genéricos que son válidos para todos los tipos de inteligencia, como, por ejemplo, la Objetividad, la Protección de las Fuentes o la Revisión Continua. Sobre ellos, la Inteligencia Táctica no presenta particularidades fundamentales. Otros, en cambio, tienen una interpretación específica en la Inteligencia Táctica o merecen resaltarse.

- El Control Centralizado pretende evitar duplicaciones e interferencias entre los distintos órganos de inteligencia, proporcionar apoyo mutuo entre los mismos y asegurar un uso económico y eficaz de los recursos. Por ello, el Mando Táctico no sólo asigna misiones a los órganos de inteligencia sino que también centraliza medios y puede prohibir determinados esfuerzos.

- La Explotación Sistemática de las Fuentes significa no sólo la asignación metódica de misiones a aquellas, sino también que las fuentes puedan utilizarse por los distintos órganos de inteligencia sin que haya barreras entre ellos.

- La Oportunidad está relacionada con la característica citada de "inmediatez". La información y, su producto elaborado, la inteligencia tienen que llegar sin retraso a los que la necesitan, siempre que sea posible en tiempo real, aunque sea como información bruta, no elaborada.

- La Accesibilidad implica que la información bruta y la inteligencia elaborada deben ser accesibles a los órganos de inteligencia de todos los escalone. Por ello, los órganos de inteligencia tienen que poder entrar en los archivos de los demás, lo que supone, hoy día, el acceso a sus sistemas informáticos. Como la cantidad de información obtenida de las fuentes va disminuyendo como consecuencia de las sucesivas redacciones de los encargados de su gestión, en ocasiones, es preciso llegar a la información original.

- La Comunicación se refiere a que el flujo de información e inteligencia entre los distintos escalones tácticos necesita ser ascendente, descendente y lateral, lo que obliga a disponer de un sistema de transmisiones rápido, fiable y redundante.

\section{El ciclo de Inteligencia}

Se llama Ciclo de Inteligencia a la secuencia de actividades por la que se obtienen informaciones, se las procesa para convertirlas en inteligencia y se pone ésta a disposición de los que la necesitan. El ciclo es un modelo único que se adapta a cada situación particular.

Las actividades del Ciclo se suelen clasificar en cuatro "fases": Dirección, Obtención, Elaboración y Difusión. En algunos ejércitos, la fase de Dirección se divide en dos, una de Planificación y otra de Dirección propiamente dicha.

Al conjunto de actividades se le denomina Ciclo por su carácter de revisión y actualización constante. Una información nueva puede provocar un cambio en el análisis de la situación lo que, a su vez, origina una nueva necesidad de inteligencia.

Sin embargo, aunque la secuencia de las fases parece indicar un orden cronológico y lineal, la realidad es que, una vez iniciado el Ciclo, la fases se solapan unas a otras, 
precisamente por la actualización constante derivada del flujo continuo de informaciones. Por eso, hoy se prefiere hablar de "Proceso", en vez de "Ciclo", proceso en el que las fases no son sucesivas sino que se influyen mutuamente, se desarrollan de forma simultánea y evolucionan constantemente.

El concepto lineal de ciclo pudiera ser aplicable a una información o noticia aislada, pero no a una situación real en la cual las informaciones llegan ininterrumpidamente. El "Ciclo de Inteligencia" es más una herramienta teórica que permite analizar y comprender el funcionamiento de un sistema de ayuda a la decisión, que un mecanismo real; es un útil pedagógico.

\subsection{Fase de dirección}

En la fase de Dirección, se determinan las necesidades de inteligencia y sus prioridades, se planifica la obtención de información, se dan órdenes a los órganos de inteligencia propios y se cursan peticiones a los de los escalones superior y laterales y se controla el funcionamiento el sistema. La característica principal de esta fase es la subsidiariedad: se asigna un trabajo de obtención a aquél que mejor puede hacerlo.

Todo el trabajo de la fase de Dirección se vierte en documentos en los que, a partir de las "necesidades" de inteligencia identificadas, se seleccionan los "indicios" que puedan responder a aquellas y, en función de estos, se determinan las "misiones" que, finalmente, se asignan a las "fuentes".

Cada vez más, a nivel táctico, el documento escrito tiende a sustituirse por un procedimiento gráfico que permite visualizar rápidamente la situación, identificar los elementos esenciales de ella y optimizar el empleo de los medios.

El proceso de creación de ese documento gráfico se conoce como INTE (Integración Terreno Enemigo) y también como IPB (Intelligence Preparation of the Battlefield). El INTE se apoyaba en una serie de dibujos o plantillas sucesivos del terreno, de la meteorología y de la doctrina y noticias del enemigo que antes se superponían para obtener así una imagen única de la situación; hoy día, se apoya en las técnicas de proceso automático de datos. Tanto en un caso como en otro, el INTE permite identificar las zonas que facilitan o dificultan la progresión de los medios propios o del enemigo, las zonas no franqueables para ciertos tipos de medios, las avenidas para los mismos y los puntos decisivos.

El INTE, que es un elemento fundamental en la fase de Dirección, es válido en todo tipo de situaciones, adaptándose, como es lógico, a cada caso concreto.

\subsection{Fase de obtención}

En la fase de Obtención, se explotan sistemáticamente todas las fuentes de información y se entrega la información obtenida a los órganos encargados de su procesamiento.

Esta fase es la más característica del ciclo de inteligencia, sin ella, no existiría la función inteligencia. A lo más, habría una actividad de asesoría al Mando.

Las fuentes son personas, cosas o acciones de las que se obtienen noticias del enemigo, del terreno, de la meteorología y del ambiente. Las fuentes pueden ser muy numerosas, utilizándose sensores terrestres, aéreos o navales, acciones de vigilancia o de reconocimiento, interceptaciones de comunicaciones y escuchas del espectro radioeléctrico, interrogatorios y 
entrevistas, examen de documentos y de material. Pero la nota distintiva de la fase de obtención, en el nivel táctico, es la "información de contacto" y de "combate" que las unidades desplegadas frente al enemigo realizan constantemente.

En ese nivel, la principal fuente de información es la actividad del enemigo o su ausencia. Son también fuentes importantes la recogida de documentos y equipos del adversario, la recogida y examen de proyectiles y de los embudos producidos por ellos y los interrogatorios del personal militar propio y del enemigo así como de la población civil. Sin embargo, esos interrogatorios serán siempre rápidos y someros y sobre la situación actual, dejando la responsabilidad de realizar interrogatorios completos a los especialistas de unidades superiores.

Todo combatiente, cualquiera que sea su función en la unidad táctica, debe tener conciencia de la necesidad de participar en la recogida de información. Los enemigos de la recogida son los despreocupados y los "coleccionistas" que guardan recuerdos.

Los sistemas de obtención de inteligencia se pueden clasificar por su origen, principalmente, en los siguientes tipos:

- HUMINT o Inteligencia clásica, obtenida por medios humanos, pudiendo utilizar éstos medios auxiliares

- IMINT o Inteligencia de Imágenes obtenida por sensores diversos, fotográfícos, térmicos, infrarrojos, radares etc.

- SIGINT o Inteligencia de Señales que engloba a COMINT y ELINT.

- COMINT o Inteligencia de Comunicaciones obtenida de escuchas o interceptaciones

- ELINT o Inteligencia Electrónica obtenida de las radiaciones electromagnéticas, distintas de las usadas en transmisiones.

Órgano de inteligencia es cualquier persona u organización dedicada a la producción de inteligencia, en cualquiera de las fases del Ciclo. Sin embargo, esta especialización de los órganos no implica que los demás elementos de una fuerza no realicen ciertas tareas de inteligencia, en especial de obtención. La Inteligencia Militar es una "Función de Combate" que obliga a todo combatiente.

La entrega de la información, así recogida, exige unas transmisiones fiables, seguras y redundantes así como el uso de formatos normalizados que permiten ahorrar tiempo y su tratamiento automático.

\subsection{Fase de elaboración}

En la fase de Elaboración, o de Procesamiento, la información obtenida se transforma en Inteligencia. Dentro de la fase hay diferentes etapas.

La primera, la "Compilación", es una actividad burocrática, de registro y organización de todo lo recibido que facilita el resto del proceso; a veces también se lleva a cabo una 
primera transcripción de la información bruta recibida, para hacerla más fácilmente procesable, como, por ejemplo, la interpretación de una grabación de un eco radar.

En la siguiente etapa, la "Valoración", se valoran las noticias por su utilidad y por su posible fiabilidad - según la valoración la noticia- y se sigue en el proceso, archivándose para una utilización futura, destruyéndola o bien enviándola un escalón superior o lateral.

Después, en la etapa de "Análisis", se analiza la noticia, se sacan deducciones y se comparan éstas con otras informaciones ya existentes.

Finalmente, la etapa siguiente es la de "Interpretación", en la que la información se transforma en inteligencia, se extraen conclusiones y se formulan hipótesis de futuro.

A nivel táctico, como ocurre en todo el Ciclo de Inteligencia, en la fase de Elaboración que, en principio parece ser una secuencia lineal de actividades, las etapas pueden simultanearse o solaparse. Por otra parte, el desarrollo de las etapas puede originar nuevas necesidades de inteligencia que confirmen o invaliden conclusiones provisionales. Además, en las unidades menores, por la tantas veces mencionada urgencia, se puede prescindir de una elaboración completa, dejando ésta a cargo de un escalón superior y trabajando solamente con información valorada. Sin embargo hay que tener en cuenta que el Jefe de la unidad lo que necesita son conclusiones no una simple colección de noticias.

\subsection{Fase de difusión}

La última fase es la de Difusión. En ella, la inteligencia es distribuida y entregada, en tiempo oportuno, en la forma y por los medios apropiados, a los que la necesitan. La difusión puede ser verbal o documental. En este último caso, los documentos pueden ser periódicos o puntuales, pero siempre deben ser claros, concisos $y$, si es posible, en formatos normalizados. Al igual que para el envío de las informaciones obtenidas, los medios de difusión deben ser fiables, redundantes y seguros, protegiendo no sólo el mensaje sino, también y fundamentalmente, la fuente. Durante el combate, el flujo de inteligencia e información será, siempre que sea posible, en tiempo real.

En resumen, a nivel táctico, la producción de inteligencia sigue el mismo patrón que en los niveles estratégico y operativo pero adaptándose el ciclo a la medida del escalón. Sus principales características son la urgencia y la limitación de medios que hacen que, muchas veces, se trabaje con la información bruta o semielaborada. Su ventaja es el contacto directo con la situación.

\section{Los retos de hov}

Desde hace unos años, los Sistemas Militares de Inteligencia y, entre ellos, el Táctico, han sufrido el impacto de dos nuevas realidades: la globalización y las nuevas tecnologías. Los sistemas han evolucionado para adaptarse a la situación, experimentando profundos cambios. El concepto fundamental de transformación de las informaciones en Inteligencia, por medio de un proceso o ciclo para ayudar al cumplimiento de una misión, continúa siendo válido. Pero han cambiado la organización, los procedimientos e incluso las doctrinas de empleo. Por supuesto, el cambio ha dado lugar a una mayor complejidad en la producción de Inteligencia. 


\subsection{La globalización}

Antes, los actores de las guerras eran los Estados y las causas de las guerras el interés, el miedo o las ideologías, o bien una mezcla de todo ello. Ahora, hay nuevos actores distintos de los Estados, como las organizaciones internacionales, grupos terroristas o subversivos, milicias paramilitares de grupos políticos e, incluso, la población civil en determinadas zonas. Y la ideología se ha convertido en la causa fundamental de la mayoría de los enfrentamientos armados.

Esa nueva situación ha dado lugar a la "Guerra Asimétrica" en la que no luchan Estados entre si, sino que los actores tradicionales se enfrentan a grupos, en la que no se desarrollan operaciones continuas entre dos ejércitos, sino episodios breves y muy violentos (caso de las Torres Gemelas) o acciones de baja intensidad continuadas (caso actual de Afganistán). En la Guerra Asimétrica, la superioridad material de las fuerzas de combate convencionales se vuelve irrelevante. Los Ejércitos no pueden abandonar sus esquemas para una guerra convencional, siempre posible, pero, tienen que tener en cuenta en sus planteamientos, cada vez más, la Guerra Asimétrica. Incluso, una guerra, en principio convencional, puede convertirse en un enfrentamiento asimétrico (caso de Irak).

La Guerra Asimétrica, como todo tipo de guerra, precisa de una Inteligencia apropiada y de uno órganos idóneos encargados de producirla y se la ha definido como "una guerra de Inteligencia y de Percepciones"

Actualmente, las Fuerzas Armadas intervienen, cada vez más, en misiones tipo Petersberg, que, en principio, no son específicamente militares (humanitarias y de evacuación; mantenimiento de la paz; gestión de crisis y operaciones de restablecimiento de la paz) y en las misiones detalladas en el Tratado de Lisboa (desarme; consejo y asistencia militar; prevención de conflictos; estabilización).

Pero como toda misión militar precisa Inteligencia para su ejecución, las misiones Petersberg confiadas a las Fuerzas Armadas la necesitan igualmente. Desde el primer momento de la planificación de una misión, aparecen las actuaciones de las Inteligencias Estratégica y Operativa; en la ejecución, están la Operativa y la Táctica; y si se llega al enfrentamiento armado, la Inteligencia Táctica cobra todo su valor. En este caso, como en todos, saber es poder y la superioridad en la Inteligencia puede suplir la inferioridad numérica.

Las intervenciones de las Fuerzas Armadas, en misiones de guerra asimétrica o Petersberg, lo son, casi siempre, en un marco multinacional. Esta circunstancia supone que, si bien el Mando es único, los Estados Mayores de la fuerza y, por ello, también los órganos de inteligencia, serán multinacionales.

El manejo de la inteligencia necesaria para la misión se enfrentará entonces a un dilema. Por una parte, habrá que compartir información con el personal de otras naciones; por otra, habrá que proteger las fuentes propias, lo que en muchos casos ocasionará problemas. Otro problema suplementario es que para que un Estado Mayor y el Ciclo de Inteligencia funcionen correctamente es preciso que exista una normalización de métodos y procedimientos, algo que la OTAN intenta conseguir con los Acuerdos de Normalización o STANAG (Standard Agreement), y la presencia de oficiales de Estado Mayor y de enlace y con conocimiento profundo de idiomas. 
En las intervenciones armadas derivadas de las misiones tipo Petersberg raramente se emplearán Grandes Unidades y lo normal será la utilización de Unidades menores. Pero a esas Unidades se les marcará la consecución de un objetivo "estratégico" definido por un órgano político, normalmente una organización internacional. Es decir que unas "unidades de nivel táctico" pasarán a realizar una misión de "nivel operativo".

Por otra parte, en un enfrentamiento asimétrico es fundamental comprender la forma de pensar y actuar del adversario y sus intenciones; es decir, hacer "Inteligencia Predictiva", algo que es característica del "Nivel Operativo". Finalmente, sensores especializados, que por su complejidad y escasez no se asignarían normalmente al nivel táctico, serán utilizados por las unidades desplegadas. De la unión de las tres circunstancias anteriores- objetivo estratégico, inteligencia predictiva y uso de material muy sofisticado- y su comparación con el tamaño de las unidades empleadas se deriva la tendencia a la fusión entre los niveles operativo y táctico, al menos en los conflictos asimétricos, así como de sus respectivas inteligencias.

En los conflictos asimétricos, la Inteligencia Táctica seguirá contando con su gran ventaja, el "contacto" con la realidad, y también con su gran inconveniente, la falta de tiempo. Por eso, será habitual que la elaboración de inteligencia se realice, en gran parte, fuera de la zona de la misión, a miles de kilómetros, en los órganos superiores de inteligencia, en el propio territorio nacional o en un Cuartel General plurinacional, en tiempo casi real, gracias a los sistemas avanzados de transmisiones.

En todo caso, en cada operación de guerra asimétrica, los órganos de inteligencia adecuados a la misión se organizan "a medida", destacándose, además, a la zona de misión equipos de escalones superiores.

\subsection{Las nuevas tecnologías}

El impacto de las nuevas tecnologías en el campo de la Inteligencia ha sido de tal repercusión que se puede decir que en la producción de Inteligencia se ha pasado de un estadio artesanal a otro industrial.

Valga, por ejemplo, la manera de realizar la "Integración terreno-enemigo" (INTE), documento gráfico antes citado. Hasta hace unos años se trabajaba con "superponibles", es decir, con dibujos sobre soportes transparentes. Estos dibujos (terreno, meteorología, plantillas con la forma de actuar del adversario) se superponían dando como resultados un gráfico con un modelo de situación. En la actualidad, el INTE se realiza fundiendo los datos en un ordenador y presentándolos sobre las pantallas de los diversos Mandos, lo que permite actualizaciones instantáneas.

El impacto de las nuevas tecnologías sobre la Inteligencia Táctica lo ha sido básicamente en tres campos: en el de los sensores, en el del tratamiento de la información y en el de las transmisiones.

-Los sensores, fijos o móviles, terrestres o sobre plataformas aéreas o navales, son cada vez mejores y más especializados. Se ha dicho que los "drones" (aviones no tripulados) y los satélites son los "ojos del Mando". Pero, por su complejidad y su coste, estos medios de obtención de inteligencia siempre serán escasos y la mayoría se situarán en niveles más elevados que el Táctico. De ahí la necesidad de que en este nivel la difusión de la información captada por los sensores sea rápida y que, incluso, tenga acceso directo a la 
misma. El acceso directo puede causar problemas de seguridad lo que obligará a establecer medidas estrictas de control.

No obstante lo anterior, debido a la incidencia de la globalización y a los enfrentamientos asimétricos, el nivel Táctico puede, en ocasiones, disponer de ciertos sensores que, normalmente serían exclusivos de niveles superiores y dada la especialización elevada, tanto del material como del personal encargado de su utilización, al nivel Táctico se le puede asignar, desde el escalón superior, un "Destacamento de Apoyo de Inteligencia".

La cantidad de información que proporcionan los sensores puede hacer difícil su interpretación y llegar a originar una "desinformación por saturación". Sin embargo, el principal problema que se presenta en el uso de sensores es el de su coordinación, en tiempo y espacio, tanto entre ellos como con las fuentes humanas, ya que ambas clases de fuentes son complementarias.

En este aspecto de coordinación a nivel Táctico es destacable el concepto ISTAR (Intelligence, Surveillance, Target Adquisition and Reconnaissance). En él, sensores técnicos y medios humanos, utilizando los métodos de Vigilancia, Adquisición de Objetivos y Reconocimiento, obtienen informaciones sobre el enemigo que se integran en un cuadro único para producir Inteligencia. El ISTAR ha dado lugar a la creación de unidades de inteligencia especializadas, - según los ejércitos de entidad compañía, batallón e incluso brigada-, dotadas de los medios técnicos necesarios. Estas unidades pueden ser agregadas, como refuerzo, a unidades de combate, como ha ocurrido, por ejemplo, en Bosnia y Afganistán.

Desde el punto de vista de organización, las unidades ISTAR no son las únicas unidades de inteligencia especializadas con nuevas tecnologías. Existen, a niveles superiores, unidades de tipo "Brigada de Inteligencia" compuestas por varios batallones equipados cada uno de ellos con sensores de distinto tipo y dedicados a un área específica - por ejemplo, unos batallones HUMINT, otros SIGINT, otros IMINT, etc-. Destacamentos de estas Brigadas se pueden asignar a unidades de nivel táctico.

- Para la Inteligencia Militar, a cualquier nivel, la finalidad de la digitalización es facilitar la acción del mando por la puesta al día de la situación, en tiempo cuasi real.

En ese sentido, la digitalización ha modificado la manera de producir inteligencia, fundamentalmente, en cuatro aspectos: la gestión automatizada de datos, la fusión de los mismos, el seguimiento de la evolución de la situación y la difusión de la inteligencia; todo ello en tiempos muy cortos.

En el tratamiento de datos, la digitalización ha posibilitado la existencia de una Base de Datos común de Inteligencia, con acceso e intercambio de ficheros a todos los niveles de Mando, el manejo de una cantidad enorme de informaciones, la recuperación y la extracción desde esa gran masa de datos de los que son verdaderamente necesarios, gracias a las técnicas de la "minería de datos ("data mining") así como la explotación inmediata de ellos a cualquier nivel.

Por otra parte, los programas informáticos de fusión de datos (como, por ejemplo, el francés FURET, de Fusión de Inteligencia Táctica) integran la inteligencia y las 
informaciones recibidas de múltiples fuentes proporcionando a los Mandos una imagen de la situación como referencia única, imagen que, además, se actualiza constantemente.

En la Inteligencia Predictiva, que el nivel Táctico precisa en los enfrentamientos asimétricos, existen los modelos heurísticos que, partiendo de una situación inicial y por medio de reglas empíricas, lógicas y probabilísticas, intentan prever la evolución con las probables reacciones del adversario en tiempo muy corto.

Todo ello hace que, como dijo el ex vicepresidente norteamericano R. Cheney, "Hoy nuestro comandantes tienen en tiempo real en la pantalla de su ordenador la situación de las fuerzas". Y Cheney se refería a las unidades tácticas hasta el escalón batallón.

Todo lo anterior, adquisición de información, difusión, acceso a ficheros, presentación de la situación, se hace posible por al extraordinaria mejoría de los sistemas de transmisiones sin la cual toda la producción de Inteligencia seguiría desarrollándose lentamente y casi en circuito cerrado, sin circulación rápida de la información. Las redes de transmisiones digitalizadas, con su gran capacidad y velocidad de transmisión, permiten que los órganos de inteligencia trabajen con las demás tecnologías. Como ejemplo del impresionante desarrollo de las comunicaciones tácticas y, dentro de ellas, de las utilizadas por los órganos de Inteligencia, basta saber que en la primera guerra de Irak, una fuerza de 500.000 hombres disponía de 100Mbits de banda ancha; unos 12 años más tarde, los 350.000 combatientes de la “Operation Irak Freedom", en la segunda guerra de Irak se apoyaban en 3.000Mbits.

Como todas las redes de transmisiones, las que se utilizan específicamente por los órganos de inteligencia tienen que estar protegidas, ser seguras y fiables y, en previsión de posibles fallos, redundantes, existiendo siempre para la comunicación entre dos puntos, además de un camino normal, al menos, una vía alternativa.

Desde el punto de vista de la Inteligencia Táctica, hay que resaltar que, hasta hace unos pocos años $\mathrm{y}$, precisamente, por la característica de urgencia en el nivel táctico, muchas veces no se podían cifrar los mensajes y había que enviarlos en claro o, como máximo, en lenguaje convenido. Hoy, los sistemas automáticos de cifrado y descifrado posibilitan la seguridad y rapidez de las comunicaciones.

\subsection{Los sistemas de sistemas}

Actualmente, los sistemas de ayuda a la decisión, de los que forman parte fundamental los órganos de inteligencia, están evolucionando hacia un "sistema de sistemas", en el que se integran en una única red sensores, decisores, plataformas de armas e inteligencia.

La finalidad de tal "sistema de sistemas" es aumentar la capacidad de acción de las fuerzas por una mejor explotación de la información. Es decir, mejorar la aplicación del poder militar por medio de la superioridad en información, transformando una ventaja en información en una ventaja decisiva en el combate, a través de la explotación oportuna de inteligencia. Ese gran "sistema" sería válido tanto para la batalla convencional como para el enfrentamiento asimétrico.

Es los Estados Unidos, el "sistema de sistemas" se denomina "Network-Centric Warfare" (NCW) o "Network-Centric Operacional". Este segundo nombre se aplica preferentemente para el caso de operaciones tipo Petersberg. En el Reino Unido, se le conoce 
como "Network Enabled Capability (NEC) y sistemas similares se llaman "Ubiquitous Command and Control (UC2) en Australia, "Network Based Defence" (NBD en Suecia), etc.

En el caso europeo uno de los sistemas más adelantados es el británico. Se apoya en tres pilares: las Redes, la Información y el Personal. En primer lugar, el NEC es una red que engloba diversas redes (10 redes especializadas que van desde la Infraestructura de Defensa a los satélites de información, desde las transmisiones en operaciones tácticas a los misiles antiaéreos) y que distribuye la información obtenida a todas las redes. En segundo lugar, NEC busca aumentar la capacidad de obtener información y de protegerla (la red que corresponde al Concepto ISTAR está incluida en este pilar). Y el tercer pilar es la selección y formación del personal capaz de comprender, conocer y utilizar el NEC

El NEC existe en los tres niveles militares: estratégico, operativo y táctico y está pensado para trabajar en operaciones conjuntas (con fuerzas de tierra, mar y aire) y combinadas (con fuerzas multinacionales, de la Alianza Atlántica o de una coalición). Incluso en el nivel Táctico, el NEC prevé que además de la integración en la Red de las fuerzas británicas y aliadas, estén incluidas en ella "otras organizaciones", como representaciones diplomáticas, ONGs, representantes de medios periodísticos o agentes de información. Esta integración de elementos dispares en la inteligencia táctica pone más en relieve la fusión progresiva de los niveles Táctico y Operacional.

En el NEC hay un enlace directo entre el nivel Estratégico y el Táctico, lo que abunda en la idea de que en las situaciones actuales de "asimetría", el nivel Táctico se convierte, muchas veces, en Operativo y que los medios tecnológicos del nivel Estratégico pueden trabajar directamente para el Táctico.

La OTAN ha adoptado la filosofía del NEC como algo común para toda la Alianza Atlántica. El sistema se llama, por ahora, NNEC. La primera N es por NATO.

Para la OTAN, el NNEC no será sólo una red, sino, sobre todo, un modo de pensar, de enfocar los problemas, una "cultura". Por ahora, la NNEC se reduce a un "trabajar juntos" y a tratar de mejorar el uso de las nuevas tecnologías, con la finalidad de usarlas en futuras misiones. El problema que se planteará es que la OTAN no tiene órganos propios de Inteligencia y que depende de los de las naciones aliadas.

\subsection{Los inconvenientes}

A pesar de las enormes y evidentes ventajas que la digitalización proporciona a la Inteligencia Militar, en sus tres niveles, también se derivan de ella, como de toda actividad humana, inconvenientes.

En principio, se pueden distinguir cuatro consecuencias negativas: el coste de los sistemas, la fragilidad de los mismos, la sobreinformación resultante y los daños a la cadena de mando.

El coste económico de la adquisición de los equipos y de su mantenimiento (realizado en gran parte por personal civil contratado directamente o perteneciente a grandes empresas, lo que plantea problemas en caso de combate), así como el de la formación del personal, crece en espiral y alcanza cifras astronómicas (el coste de un solo proyecto de información táctica del ejército americano se estima en unos 12.000 millones de dólares). Sólo las grandes potencias pueden permitirse tales programas que, además, tardan años en ser operativos. Las 
potencias medias y pequeñas tendrán que resignarse a poseer sólo algunos sistemas menores $\mathrm{y}$, por ello, resignarse al papel de fuerzas auxiliares.

La fragilidad de los nuevos sistemas digitales se deriva de la dependencia tecnológica del extranjero, del empleo de técnicos civiles en operaciones de mantenimiento y reparación y de su sensibilidad ante ataques cibernéticos. El daño en combate o por avería de algún elemento de la red obligaría a que ésta trabajase en "modo degradado", lo que reduciría su eficacia. Por otra parte, en la guerra asimétrica, un enemigo "en alpargatas", que utilice técnicas de combate primitivas, que conozca perfectamente el terreno y el ambiente y que esté fuertemente motivado por motivos ideológicos puede dañar seriamente los complicados sistemas tecnológicos como el NEC sin ni siquiera aparecer en la pantalla del ordenador del Mando

El enorme flujo de información recibida a través de las redes digitales, aunque llegue depurado y junto con inteligencia ya elaborada, puede, por una parte, saturar el sistema y, por otra, producir indecisión en el Mando ante el cúmulo de noticias y su rápida variación

El flujo de información y el acceso al mismo a todos los niveles, puede ocasionar que un Jefe táctico, que está viendo en la pantalla de su ordenador la imagen de la situación, caiga en la tentación de tomar una decisión que correspondería a un escalón subordinado. ( por ejemplo, el General de la División que ordena el contraataque de una compañía de reserva). Esa actitud dañaría el espíritu de iniciativa de los mandos inferiores y anularía su libertad para decidir como cumplir la misión.

El caso contrario, el del subordinado que utiliza su acceso las redes para dirigirse directamente a escalones superiores, para exponer sus ideas o proponer medidas, saltándose la vía reglamentaria, también puede darse. Esta actitud, en la milicia se llama indisciplina y la indisciplina deshace los ejércitos.

También puede darse que haya errores de interpretación de la "realidad virtual" que aparece en pantalla y, en consecuencia, un desconocimiento de la realidad "real". Nada indica que un moderno general, sentado frente a su ordenador, pueda interpretar la realidad y decidir en consecuencia mejor que uno de siglos pasados, que a caballo, sobre una colina, mirando por un anteojo, veía la realidad. Finalmente, el hombre es el sistema y es, a la vez, el eslabón más débil y el fundamental de la cadena de Mando y de Inteligencia.

\section{REFERENCIAS}

- Kent, Sherman: Inteligencia Estratégica, Buenos Aires, Pleamar, 1989.

- Vinuesa, Arturo: La Inteligencia Estratégica, Ruptura Gráfica, Madrid,1995.

- Estudio de "Inteligencia Operacional". Cuaderno de Estrategia 31. Instituto Español de Estudios Estratégicos. Madrid, 1991

- Estudios sob re Inteligencia. - Cuaderno de Estrategia 127. Instituto Español de Estudios Estratégicos. Madrid, 2004.

- El papel de la Inteligencia ante los retos de la seguridad y la defensa nacional, Cuaderno de Estrategia 130. Instituto Español de Estudios Estratégicos. Madrid, 2005. 
- Reglamentos sobre Inteligencia del Estado Mayor de la Defensa y del Ejército de Tierra .

- NATO Glossary of Terms and Definitions (AAP-6).

- Publicaciones “Objectif Doctrine” y "Doctrine” del Ejército Francés.

- Manuales de Campaña del Ejército Norteamericano, en particular las series FM.2 y FM.34

- Joint and Nacional Intelligence Support to Military Operations. JP 2-01

- Manual SOA sobre Inteligencia de la "Escuela de las Américas".

- Publicaciones del Ministerio de Defensa Británico “JDP”, en particular la JDP-0-01 y "Understanding NEC". 